\title{
Indicateurs du développement durable des transports et concertation avec le public : postures stratégiques et argumentations
}

\author{
Christophe Bouni ${ }^{1}$, Gaëlle Chevillotte ${ }^{2}$, Agathe Dufour ${ }^{3}$ \\ 1 Économiste, Applications des Sciences de l'Action (AScA), 8 rue Legouvé, 75010 Paris, France \\ 2 Ingénieur du génie rural des eaux et forêts, Applications des Sciences de l'Action (AScA), 8 rue Legouvé, 75010 Paris, France \\ 3 Juriste, Applications des Sciences de l'Action (AScA), 8 rue Legouvé, 75010 Paris, France
}

L'élaboration d'indicateurs du développement durable afin d'éclairer les décisions publiques est désormais une obligation fixée par la loi. Pour les entreprises, ces indicateurs doivent servir à optimiser la production et les services ; encore faut-il définir de quel développement durable il s'agit et quelles responsabilités les entreprises acceptent d'endosser. Les indicateurs de développement durable peuvent en effet servir au calcul d'impact afin de remédier aux dommages environnementaux, mais ils peuvent également servir d'instruments de négociation entre les différentes parties prenantes et dépasser les approches sectorielles pour mettre en question les modes de vie. Différentes déclinaisons du développement durable émergent ici à partir d'expériences de concertation sur ce que devrait être une «bonne» politique de transports.

La Rédaction

\section{Mots-clés :}

développement durable ; politiques de transport ; indicateurs ; gouvernance

\section{Keywords: Sustainable development; transport policies; indicators; governance}

Résumé - Réalisé sur la base de l'analyse d'expériences françaises, étrangères et internationales d'indicateurs du développement durable des transports, ce travail montre la pertinence d'une lecture des outils proposés, fondée sur la recherche des postures argumentatives qui les sous-tendent. Trois postures sont ainsi repérables au sein de ces divers travaux : une posture intégratrice visant à l'internalisation des effets externes des transports ; une posture critique, d'inspiration environnementale, interpellant le monde des transports à l'aide de critères externes à ses préoccupations premières ; enfin, une posture globalisante et désincarnée, abordant les transports au travers d'une approche de développement durable d'une nation. L'intérêt analytique de ces trois postures a été confirmé par la mise en évidence des arguments échangés à l'occasion de quatre expériences de participation du public. En conclusion, l'article appelle à mieux définir les modes de gouvernance auxquels s'adressent les expériences d'indicateurs, afin que ces derniers participent plus activement à l'enrichissement des débats portant sur ces politiques publiques structurantes.

Auteur correspondant :

Christophe Bouni, christophe.bouni@asca-net.com

AScA : http://www.asca-net.com 


\begin{abstract}
different development aspects, transport being only one of their concerns. We were able to demonstrate the analytical interest of these three postures in a study of four experiences of public debate. To conclude, our paper calls for a better definition of the governance contexts in which the sustainable transport indicators are used. The indicators proposed in the experiences studied relate to different postures within the same framework of debates and are difficult to use in describing them. Working on the governance context is essential to ensure a proper use of indicators that will enrich concertation and debates among actors.
\end{abstract}

\title{
Introduction
}

Le travail présenté ici s'inscrit dans les travaux du Programme national de recherche et d'innovation dans les transports terrestres (PREDIT, groupe 11) $)^{1}$. Au sein des différentes thématiques proposées par ce programme, la question de l'efficacité des politiques de transport est présentée de manière transversale au travers des questions d'évaluation de politique publique, de prospective ou encore de participation du public. L'objectif même de ces politiques est questionné à cette occasion, dans la mesure où les critères de jugement se sont progressivement multipliés ces dernières décennies sous la double poussée des considérations liées au développement durable et de l'essor de procédures de concertation et de débat public.

Traditionnellement, la croissance de l'offre de transport est légitimée et rendue souhaitable dans la perspective de favoriser la mobilité des personnes et des biens, de réduire toujours plus les distances entre les hommes et leurs productions, afin de minimiser les contraintes de l'espace et du temps. Une meilleure vie est ainsi assurée par davantage de transports (plus rapides, plus confortables), et ces discours classiques s'appuient notamment sur les liens qu'entretiennent le développement des transports et la croissance économique (CEMT, 2005).

D'autres considérants sont cependant apparus, questionnant les transports sur le plan des contraintes que leur développement induit sur l'environnement naturel, les paysages, la santé des personnes, mais également sur la structuration des territoires, les phénomènes de concentration ou de mitage et, au-delà, sur les modalités de développement de nos sociétés.

$\mathrm{Au}$ fur et à mesure de l'ouverture de la politique des transports à ces considérations préalablement jugées « externes », la question de l'échelle d'analyse prend toute son acuité. La norme de jugement des transports évolue et se complexifie et, concomitamment, le sens à donner à ces questions d'efficacité d'une politique de transports. On en vient finalement à se poser la question de ce que pourrait bien être une «bonne» politique des transports :

\footnotetext{
${ }^{1}$ Le PREDIT est un programme national initié et conduit par les ministères chargés de la recherche, des transports, de l'environnement et de l'industrie, l'ADEME et OSEO. Ce travail a été financé par le PREDIT et a également bénéficié d'une participation financière de Réseau ferré de France.
}

- une politique permettant d'assurer la meilleure desserte possible, là où les besoins se font sentir (« mettre en place une offre de transport en adéquation avec une demande de mobilité »)?

- une politique permettant de canaliser la demande de mobilité et de minimiser les impacts négatifs des équipements de transport et des déplacements du point de vue des ressources et des pollutions ( « réguler la demande de transports et minimiser les impacts de la mobilité »)?

- une politique intégrée, proposant la maximisation des services et la minimisation des coûts qui leur sont associés («développer l'offre de transport et limiter les nuisances et les coûts induits par ces activités »)?

On conçoit aisément que les questions d'efficacité des politiques de transport se posent de manières différentes selon que l'on revendique l'une ou l'autre des postures proposées ci-dessus, ou toutes combinaisons de ces finalités. Nous proposons ici une réflexion portant sur ces dimensions d'efficacité et, plus généralement, de jugement des politiques de transport.

Pour rendre compte des efforts en faveur de l'analyse et de l'évaluation des politiques, nous avons choisi de nous intéresser, d'une part, aux propositions de structuration d'indicateurs dans ce secteur de politique publique et, d'autre part, aux arguments échangés lors de concertations et de débats publics portant sur d'importants projets d'infrastructures de transport. Si les systèmes d'indicateurs constituent un traditionnel outil d'évaluation technique, indépendant des procédures de conception de ces projets, nous postulons que les délibérations publiques récemment organisées dans le respect des nouvelles réglementations contribuent à fonder une nouvelle forme d'évaluation participative dont les enseignements sont aujourd'hui sous-estimés. Notre hypothèse consiste ainsi à préjuger d'un lien à expliciter entre les questionnements évaluatifs portés sur les transports par les systèmes d'indicateurs développés de par le monde et le contenu des arguments échangés lors des débats, à l'occasion de projets d'infrastructures.

De nombreux pays, des organismes internationaux ont produit durant la dernière décennie une quantité importante de rapports d'état des transports, mobilisant tableaux de bord et descriptifs des politiques menées. 
Face à la richesse et à la diversité des documents, nous avons retenu une classification de ces travaux selon une typologie de sens plus que de connaissance. Elle identifie trois cadres types (trois postures) pour la construction des indicateurs, axés sur des postures stratégiques différentes pour répondre aux enjeux de l'évolution actuelle des politiques de transport. Parallèlement, quatre cas de concertation et de débats publics autour de projets d'infrastructures ferroviaires ont été étudiés, afin d'identifier les indicateurs sous-jacents aux arguments énoncés par le public et de confronter ces « indicateurs de la parole» aux indicateurs issus de l'analyse bibliographique.

Cette démarche nous conduit à présenter tout d'abord une synthèse des réflexions produites ces dernières années en matière d'évaluation des politiques de transport. Sur cette base, les trois postures stratégiques recensées sont ensuite exposées pour permettre l'analyse des expériences de conception d'indicateurs. Les arguments échangés au cours des procédures publiques étudiées sont ensuite présentés au regard de cette problématique d'indicateurs du développement durable. Enfin, nous concluons cet article sur les enjeux de gouvernance que suscitent ces résultats.

\section{Une importante réflexion sur l'évaluation des politiques de transport... encore inachevée}

Le travail a consisté à rassembler une bibliographie concernant l'évaluation de la politique des transports, laquelle s'est avérée particulièrement large. Nous avons essentiellement utilisé les sites Internet des principaux organismes internationaux - OCDE, AEE et Nations unies -, des laboratoires de recherche français tels que le Laboratoire d'économie des transports (LET) ou l'Institut national de recherche sur les transports et leur sécurité (INRETS). Nous avons également choisi de nous intéresser aux expériences menées par certains pays, soit parce qu'elles étaient susceptibles d'apporter une vision quelque peu élargie par rapport aux expériences conduites en Europe (Australie et Nouvelle-Zélande), soit parce qu'elles étaient elles-mêmes citées par d'autres. Enfin, deux documents nous ont fourni un certain nombre de pistes quant aux expériences pouvant être considérées comme incontournables : il s'agit de Verry et Nicolas (2005) et de Rahman et Van Grol (2005). Nous avons ainsi pu répertorier quelques axes fondamentaux de questionnement.

\section{Relever le défi d'une gestion de la demande de mobilité}

Le niveau international est particulièrement révélateur de cette abondance bibliographique. Ainsi, durant 50 ans, la Conférence européenne des ministres des transports
$(\mathrm{CEMT})^{2}$ a produit plus de 250 documents. Face à cette profusion, elle a elle-même souhaité, en 2003, entamer un exercice de vérification quant à la pertinence de ces différents textes, notamment au regard des évolutions contextuelles (CEM, 2003a). Elle a par ailleurs produit différents rapports revenant sur ces 50 années de politique des transports afin d'en mesurer les succès, mais aussi d'en dévoiler les échecs et de déterminer les défis qui se font jour sur la base de l'expérience acquise et des nouveaux enjeux. Ainsi, si des réussites sont enregistrées dans le secteur des transports en termes, notamment, de gains de productivité ayant contribué à la croissance économique, des insuffisances manifestes sont soulignées concernant plus particulièrement les instruments politiques, jugés limités et mettant un accent exagéré sur l'offre et l'aide aux producteurs, alors même que les structures institutionnelles qui encadrent le secteur demeurent trop fragmentées. Par ailleurs, la croissance prévue du trafic ne pourra être gérée selon les méthodes traditionnelles consistant à accroître la capacité infrastructurelle. Selon la CEMT, il convient donc de mettre en place de nouvelles incitations et de nouveaux instruments de tarification, d'investissement et de gestion de la demande (CEMT, 2003b).

\section{Se justifier face aux enjeux du développement durable}

Outre ces éléments concernant l'offre et la demande de transport, la dernière décennie a été marquée par la montée de questionnements sur les conséquences du développement des transports sur l'environnement et, plus généralement, la société, dans une perspective de développement durable. Cette interrogation a notamment pris la forme d'expériences relatives à la mise en place d'indicateurs. Au regard de notre problématique, nous nous sommes tout particulièrement intéressés à l'analyse de la manière dont ces expériences traduisent une volonté d'ouverture d'un secteur vers une ou plusieurs autres dimensions du monde qui l'entoure. On peut, en effet, constater la variété des types d'indicateurs produits. Divers travaux internationaux (OCDE notamment) prônent, par exemple, le recours à des éléments d'évaluation des politiques de transport au travers $\mathrm{d}$ 'indicateurs d'environnement, de performance et de développement durable examinant les politiques de transport dans une perspective globale et transversale. Le concept de développement

\footnotetext{
2 Organisation intergouvernementale créée par un protocole signé à Bruxelles le 17 octobre 1953 et visant à constituer un forum de coopération politique au service des ministres responsables du secteur des transports, et plus particulièrement des transports terrestres. La CEMT réunit 41 pays membres, 6 pays ayant un statut de membre associé (Australie, Canada, États-Unis, Japon, Nouvelle-Zélande et République de Corée) et 2 pays observateurs (Arménie et Maroc).
} 
durable est ainsi décliné par ces expérimentations en s'appuyant sur les trois piliers (environnement, social et économie) et sur divers principes dont l'équité ou l'efficacité. Face à cette diversité d'indicateurs, la question de leur utilisation alternative ou complémentaire se pose. Il est ainsi difficile de repérer des expériences concrètes, hormis quelques expérimentations ponctuelles concernant, par exemple, l'évaluation de la performance des réseaux routiers, les modes de déplacement à l'échelle des aires urbaines, l'évolution des normes, notamment d'émissions ou, plus généralement, de qualités techniques des véhicules. Si ces expériences d'indicateurs du développement durable sont inspirées pour la plupart de démarches internationales, elles peinent à trouver une application généralisée.

De nombreuses questions restent sans réponse dans la littérature actuelle. Doit-on structurer un ensemble d'indicateurs en fonction d'une seule logique de construction? Peut-on associer au sein d'un même système des indicateurs de différents types? Quels sont alors les objectifs que l'on peut assigner à ces différentes combinaisons? Comment favoriser l'utilisation concrète de ces outils? Enfin, et surtout, quelles sont les implications attendues de l'utilisation de ces divers outils de connaissance et/ou d'évaluation sur les finalités de la politique des transports?

\section{Et si le monde des transports ne se suffisait plus à lui-même}

En écho aux interrogations formulées précédemment, nous avons également repéré, au cours de notre recherche bibliographique, un certain nombre de documents - issus pour la plupart d'organismes internationaux - traitant davantage des liens entre instruments (existants, à renforcer ou à créer) et décision en matière de politique des transports ou, plus largement, de développement durable. Ces documents, pour la plupart, prônent à la fois le recours à une meilleure pratique des analyses coûts/bénéfices, mais encouragent également les pouvoirs publics à recourir à l'évaluation environnementale stratégique « en l'intégrant pleinement dans les processus de décision stratégique pour qu'elle donne tous ses résultats. Elle doit pour cela être rattachée à chaque phase du processus de planification qui conduit à une décision... » (CEMT, 1998).

La Commission du développement durable du Conseil économique et social des Nations unies a également consacré certaines de ses réflexions à la prise de décision (CDD, 2001a), constatant que « la réalisation des objectifs visant le développement durable exige la mise au point de nouvelles modalités pour la prise de décisions de politique générale ». Dès lors, elle formule des recommandations visant notamment à modifier les cadres institutionnels et juridiques, à formuler des stratégies intégrées de développement durable à l'échelle nationale et à améliorer les systèmes d'information à l'appui de la prise de décisions. Plus spécifiquement pour le secteur des transports, la Commission s'est penchée sur les différentes actions entreprises par la Commission économique pour l'Europe de l'ONU, afin de réguler l'activité des transports en tenant compte à la fois de sa contribution au développement économique et social et de ses impacts sur l'environnement et la santé (CDD, 2001b). Dans ce document, la Commission préconise la mise en œuvre de solutions cohérentes, intégrées et de long terme, favorisant l'engagement et la participation de tous les acteurs pertinents aux niveaux international, national, régional et local. Enfin, parmi les dix lignes directrices proposées par l'OCDE pour des transports écologiquement viables (OCDE, 2003), la dernière insiste sur la nécessité d'impliquer les parties concernées, de s'assurer de leur appui et de leur engagement et de permettre la participation du grand public.

Ce tour d'horizon bibliographique nous conduit finalement à penser que le monde des transports se trouve bien à un moment charnière de son évolution. Après avoir traversé la deuxième partie du siècle dernier dans la conviction de participer activement à l'évolution de la société, sans que la question de sa légitimité ne semble s'être posée, la politique des transports est aujourd'hui à la recherche d'un nouveau souffle. L'évolution du contexte international des années $1990^{3}$ ouvre la voie à une nécessaire hiérarchisation des enjeux du développement. Comme d'autres infrastructures structurantes, le monde des transports doit aujourd'hui développer des outils lui permettant de légitimer son rôle dans la société. Les plus récentes expériences recensées au cours de notre recherche semblent indiquer que le secteur des politiques de transport s'interroge effectivement sur les modes de planification et de prise de décision susceptibles d'élargir le cercle de la consultation, afin de préserver et de renforcer sa légitimité.

La partie suivante met ainsi en évidence trois cadres types, polarisant ces efforts en vue de construire des systèmes d'indicateurs élargissant la base de légitimité de la politique des transports.

\section{Trois postures pour la construction d'indicateurs de développement durable des transports}

L'analyse bibliographique dont il est question ici s'est focalisée sur les expériences d'élaboration de systèmes $\mathrm{d}$ 'indicateurs de développement durable dans le domaine des transports. Dans la plupart des cas, les documents étudiés s'inscrivent dans une philosophie générale faisant référence aux grands principes et objectifs du développement durable tels que définis, par exemple, dans le rapport

\footnotetext{
${ }^{3}$ Rio, 1992-Kyoto, 1997.
} 
Brundtland de 1987. Pour aller au-delà de ces objectifs généraux, trois postures issues de l'analyse bibliographique constituent des cadres types à l'aide desquels on peut analyser les processus de construction des systèmes d'indicateurs.

\section{Une posture intégrative}

Une première posture rassemble les expériences qui visent à internaliser les effets externes des transports sur les trois dimensions du développement durable. Ces expériences se placent du point de vue du gestionnaire, du porteur de projet des politiques de transport, et cherchent à ouvrir le secteur du transport au développement durable (avec comme référence, en général, le rapport Brundtland). Ce faisant, elles tendent à accroître la légitimité des politiques de transport en responsabilisant le secteur visà-vis des problématiques environnementales et sociales auxquelles il est confronté. Il s'agit alors de systèmes d'indicateurs dont la finalité est l'expansion du champ maîtrisé par le monde des transports. Dans ce cadre, et en mobilisant les méthodes et les moyens éprouvés qui sont les siens, le monde des transports se soumet avec un succès annoncé à l'épreuve de l'intégration de nouvelles dimensions au sein de son propre système de hiérarchie de valeurs.

Plusieurs expériences peuvent illustrer cette posture. Citons le projet SIMBAD (Verry et Nicolas, 2005) et ses indicateurs de mobilité durable : indicateur de consommation d'espace par les transports, à minimiser ; indicateur d'accessibilité à l'emploi, à maximiser ; indicateur d'accessibilité sociale, avec la distance quotidienne parcourue. Autre exemple : How to Monitor Indicators in Local Transport Plans and Annual Progress Reports (Transport Statistics Personal Travel UK, 2004), qui propose des indicateurs de coûts/efficacité.

Il est intéressant de constater que de telles expérimentations visent des acteurs locaux du monde des transports, pour lesquels elles fournissent des outils de gestion intégrés pouvant permettre de s'affranchir d'approches plus procédurales faisant appel à un ensemble de parties intéressées.

D'autres expériences plus globales, menées par l'OCDE et visant explicitement à mesurer la performance des transports, voire leurs avantages économiques, illustrent également cette approche (OCDE, 2001a et b). Il s'agit cette fois de fournir des éléments permettant l'évaluation des progrès des politiques de transport au regard de leurs finalités ou du respect des contraintes qui les encadrent. Quinze indicateurs sont proposés, dont le niveau de satisfaction relatif au temps de parcours, à la fiabilité, à l'état des routes, ou encore le niveau des risques et le montant des programmes pour l'environnement.
Ce qui est le plus souvent recherché à travers la mise en place d'indicateurs, dans le cadre de cette approche, est l'optimisation des politiques de transport. La comparaison de différents scénarios d'optimisation des services (économiques et sociaux) sous contraintes de coûts (environnementaux et sociaux) est souvent utilisée pour cela. L'approche est donc, en général, peu normative : les normes utilisées sont évolutives, elles se définissent en comparaison plutôt qu'a priori. Par ailleurs, cette approche s'inscrit le plus souvent, sans que cela soit explicité clairement, dans un cadre de soutenabilité faible, induisant la commensurabilité des éléments chiffrés.

L'analyse de ces expériences d'élaboration d'indicateurs de développement durable renvoie à différentes questions portant sur les limites et les potentialités du secteur des transports à s'élargir et à intégrer, de son point de vue, les dimensions du développement durable : quelle est la marge potentielle d'élargissement du champ des politiques de transport? Y a-il des limites à cet élargissement et à cette intégration? Quelles sont les modalités de gouvernance qui permettent de les établir?

\section{Une posture critique}

Un deuxième type d'expériences aborde la question des indicateurs dans le domaine des transports d'un point de vue externe, le plus souvent environnemental. L'exemple type qui illustre cette posture est le travail sur les indicateurs environnementaux appliqués aux transports mené par l'OCDE depuis 1990, ou encore le projet $\mathrm{TERM}^{4}$, lancé en 1998 par l'Agence européenne de l'environnement (AEE, 2000) et qui donne lieu à un rapport annuel. Les systèmes d'indicateurs proposés ont pour objectif de constater les débordements et les excès du marché des transports, d'illustrer les problèmes posés ou d'appeler au respect plus ou moins strict de normes le plus souvent externes au secteur des transports (consommation d'énergie par tonne par kilomètre ou par personne au kilomètre, émission de divers polluants, exposition au bruit...). Ces expériences sont soit purement environnementalistes, soit ouvertes sur d'autres dimensions : sociales, comportementales ( $c f$. par exemple PROPOLIS, 2004), suivant la même logique. Les approches purement environnementalistes, relativement anciennes (années 1990), reposent souvent sur l'utilisation des modèles Pression-État-Réponse (PER) et Driving forces-Pressure-State-Impact-Response (DPSIR), qui sont traditionnellement reconnus comme adaptés pour traiter des problématiques environnementales. Ces expériences sont par ailleurs axées, pour la plupart, sur une logique de mesure de la performance soit dans un cadre normatif, soit dans le cadre de suivi de tendances ou de comparaisons de politiques de transport. Elles doivent

\footnotetext{
4 TERM: Transport and Environment Reporting Mechanism (Bilan indiciaire sur les transports et l'environnement).
} 
permettre de répondre à la question des conséquences des politiques de transport sur l'environnement et à celle des évolutions nécessaires pour atteindre des normes environnementales. Une expérience internationale se démarque plus particulièrement dans cette catégorie. Il s'agit du projet «TEV : transports écologiquement viables », lancé fin 1994 par la commission Transport du Comité des politiques d'environnement de l'OCDE, qui propose pour la première fois dans ce domaine d'étudier l'évolution des transports via une approche prospective utilisant des scénarios «à rebours »(OCDE, 2001c). Enfin, notons deux expériences qui peuvent être qualifiées de recherche d'empreintes écologiques, l'une développée par Jens Borken (2003) au sein de l'institut de recherche allemand sur les transports (Institut für Verkehrsforschung) et celle élaborée par le Stockholm Environment Institute (Barret et al., 2001).

L'analyse de ces expériences questionne également, comme dans le premier cas, la légitimité des politiques de transport, mais cette fois-ci du point de vue de la défense de l'environnement, voire du social : jusqu'où le monde des transports peut-il décider lui-même de la manière $\mathrm{d}$ 'intégrer et de prendre en compte les autres dimensions du développement durable? Que doit-il intégrer, avec qui, sous quels modes (gouvernance, négociation) ? Contrairement à la précédente, cette posture, résolument critique, des systèmes d'indicateurs, laisse supposer un nécessaire changement dans la composition et la structure du collectif des acteurs impliqués dans l'élaboration des politiques de transport pour prendre en compte les nouvelles normes venues de l'extérieur. Cependant, les expériences étudiées ici ne prévoient pas de manière explicite les conditions d'utilisation des systèmes d'indicateurs qu'elles ont produits. Autrement dit, elles ne traitent pas de la question de la capacité du collectif des acteurs du transport à intégrer ces nouvelles normes ni même à promouvoir le débat autour de celles-ci. Les indicateurs sont ainsi construits pour structurer la critique et non pas les débats.

\section{Une posture globale}

Enfin, une troisième série d'expériences propose une approche qui cherche à illustrer la complexité de la notion de développement durable en révélant la multiplicité des interfaces entre les trois dimensions. Dans ces expériences, le lien avec le caractère opérationnel des politiques de transport est faible, ces dernières ne sont d'ailleurs pas toujours au centre des systèmes d'indicateurs. Par ailleurs, le point de vue à partir duquel on se place n'est pas explicite. De ce fait, la question de l'efficacité des politiques de transport ne se pose pas clairement, le niveau d'analyse étant en effet trop éloigné de l'action et trop désincarné. Il semble que ces systèmes d'indicateurs visent plus à prendre du recul sur les dynamiques de développement de nos sociétés, en s'adressant aux citoyens, aux individus, qu'à engendrer l'action ou la critique en ciblant des acteurs impliqués professionnellement ou engagés civilement.

On retrouve ici les expériences nationales issues des démarches internationales (programme de travail portant sur les indicateurs de développement durable conduit par la CDD depuis 1995 et ayant abouti à la publication, en 2001, d'un guide méthodologique à destination des décideurs politiques nationaux pour la mise en place de tels indicateurs : CDD, 2001c), qui visent à bâtir des indicateurs de développement durable nationaux : la thématique du transport y est présente mais pas plus qu'une autre et se situe à une échelle globale. Par exemple, la stratégie du Royaume-Uni cite le PNB par kilomètre parcouru par les poids lourds, les modes de transport des enfants de 5 à 16 ans (à pieds, en vélo, en voiture, en bus...), la distance de déplacement par catégorie de revenu, etc. Du fait de l'aspect désincarné et peu lié à l'action de ces expériences, les indicateurs de développement durable couvrent un large champ, la seule clé de tri ou de hiérarchisation, plus ou moins explicite, étant la faisabilité d'instruction. C'est dans ce type d'expériences, d'ailleurs, que l'on peut rencontrer des approches « procédurales » ou « consensuelles » qui font appel à la participation d'acteurs externes au secteur des transports.

L'analyse des expériences de posture 3 pose de manière particulièrement accrue la question du lien entre production et utilisation des indicateurs. Alors que, pour les postures 1 et 2 , ce lien est plus évident : gestion intégrée des nouvelles dimensions (posture 1), structuration de la critique du système des transports (posture 2), dans le cas de la posture 3, il n'est pas explicité dans les expériences à l'échelle nationale. Ces expériences visent en effet davantage à révéler les contradictions ou les synergies entre les différentes dimensions qu'à orienter l'action ou organiser la critique. Rien ne préjuge de l'utilisation qui peut être faite des indicateurs produits : qui peut utiliser ces indicateurs, avec quelle légitimité, quelles procédures?

\section{La concertation et le débat public comme scènes de mobilisation des indicateurs}

Si l'on cherche maintenant à représenter ces postures au sein d'une vision d'ensemble de la politique des transports, on perçoit ces systèmes $d$ 'indicateurs comme autant de tentatives de donner un corps aux liens (ou plus certainement à certains des liens) qui unissent les politiques de transport aux trois piliers que sont l'économie, l'environnement et le social. L'enjeu est ici de définir les conditions nécessaires à la vie des indicateurs, ce qui conduit à s'interroger sur leur utilité.

Les théories portant sur les indicateurs affirment notamment que ces outils doivent fournir une information appropriée aux besoins de leurs destinataires, compte tenu de l'état des connaissances scientifiques. La définition 
d'un indicateur repose alors sur de nombreux choix concernant les objectifs, les modèles scientifiques sousjacents, la forme définitive, les utilisateurs visés... Il s'ensuit qu'une part importante de subjectivité est nécessairement impliquée dans la définition d'indicateurs, car les critères déterminants de leur construction ne peuvent être exclusivement fondés sur des règles scientifiques couramment acceptées. Par là même, les indicateurs dépassent la simple mesure statistique descriptive pour constituer une interprétation de la réalité qui prend la forme d'un modèle (Braat, 1991), «compromis entre des résultats scientifiques et la demande $\mathrm{d}^{\prime}$ information concise des utilisateurs » (Verbruggen et Kuik, 1991).

Cette subjectivité dans la production des indicateurs conduit à focaliser l'attention sur les conditions procédurales et institutionnelles de leur utilisation. C'est à ce titre que nous avons choisi de nous intéresser aux concertations et débats publics en tant que scènes potentielles de mobilisation d'indicateurs. Quatre cas de concertation ou de débat public portant sur des projets d'infrastructures ferroviaires ont ainsi été analysés, sur la base notamment des minutes retranscrites des réunions (Encadré). Les arguments du public ont été systématiquement repérés et, pour chacun d'entre eux, ont été notées les thématiques en jeu, les problématiques soulignées, les informations mobilisées ou qui auraient pu l'être pour étayer l'argument, enfin les normes sous-jacentes à ces expressions. Nous avons considéré les arguments échangés lors des débats comme autant d'occasions de proposer des indicateurs pour juger des situations discutées. Ce travail nous a permis de concevoir, pour les quatre cas analysés, des systèmes virtuels d'indicateurs justifiés par les arguments échangés et potentiellement utilisables pour l'évaluation de ces infrastructures. La base de données réalisée a permis de retenir 412 arguments issus des débats et porteurs d'un enjeu de conception d'indicateurs (sur un peu plus de 700 arguments recensés). Nous présentons ici les principaux résultats de l'analyse de cette base de données au regard des trois postures présentées précédemment.

Un premier résultat de cette analyse a trait à l'utilisation systématique de la posture critique pour aborder les questions relatives aux thématiques des dommages environnementaux et de l'exposition aux risques : par exemple, l'évolution cartographiée de la population exposée aux risques (accidents, risques industriels et environnementaux), ou encore les impacts environnementaux du projet y compris durant la phase de travaux. Les projets sont, en effet, uniquement perçus comme des facteurs $d$ 'intensification des nuisances ou des aléas. Il n'est donc pas envisagé qu'un projet de ce type, quel que soit le contexte, puisse participer à une meilleure gestion des risques ou de l'environnement. Le traitement de ces thématiques se situe sur un plan éminemment conflictuel, portant à concevoir des indicateurs spécifiques à prendre en considération au sein d'analyses multicritères et non pas intégrées.

\section{Encadré. Quatre cas de concertation ou de débat public}

- Contournement ferroviaire Nîmes-Montpellier

Il s'agit d'une ligne nouvelle de $70 \mathrm{~km}$ entre Nîmes et Montpellier, se raccordant au réseau de la LGV Méditerranée à l'est et à la ligne Perpignan-Barcelone à l'ouest. Sa finalité est double : fret et voyageurs.

Le dispositif de concertation a été mis en place de juin à juillet 2003.

- Ligne fret Lyon-Turin

La concertation porte sur les différents tracés envisageables entre Ambérieu-en-Bugey et Saint-Jean-de-Maurienne, soit la partie française du projet de ligne fret.

Le dispositif de concertation étudié s'étend sur deux mois et demi à l'automne 2003.

- Liaison ferroviaire entre Paris et l'aéroport de RoissyCharles de Gaulle : CDG Express

Projet d'une ligne ferroviaire dédiée aux voyageurs aériens entre Paris et Roissy.

Débat public CNDP (Commission national du débat public) entre le 25 août et le 15 décembre 2003.

- Suppression du bouchon ferroviaire de Bordeaux

Il s'agit de la création d'une gare multimodale et d'une nouvelle traversée de la Garonne, en raison de l'encombrement actuel et de la future ligne TGV Sud Europe Atlantique.

Concertation préalable à l'enquête publique durant 4 mois, de fin 2002 à fin mars 2003.

Il s'ensuit que la négociation est le cadre d'échange probablement mobilisé pour le traitement de ces thématiques. Les compensations sont attendues de la part du maître d'ouvrage et devront faire appel à d'autres dimensions que celles des problèmes rencontrés. L'expropriation, la compensation financière, les aménagements connexes sont ainsi sollicités comme susceptibles d'apporter un apaisement.

En revanche, de nombreuses thématiques issues des débats interpellent les aménageurs pour la prise en considération de diverses problématiques induites par le projet, se référant ainsi à la première posture d'intégration. Il s'agit de l'intégralité des questions portant sur les thématiques suivantes : " articulation des dimensions temporelles (mesure de la durée de conception du projet de l'annonce au public à la mise en service, impact de l'ajournement des projets locaux d'intérêt général), «qualité et cadre de vie », «bruit», «variations du prix de l'immobilier », « effets de coupure», «entretien et sauvegarde du patrimoine existant », «droit du travail » (pourcentage des ouvriers ayant retrouvé du travail à la fin du chantier), «gestion financière " (coût au kilomètre parcouru). On retrouve également cette posture au sein d'une moitié des questionnements portant sur la thématique «équité, répartition ». Pour toutes ces thématiques, les expressions relèvent souvent de préoccupations locales, dont le traitement peut être réalisé par une prise en considération au sein même du projet, sans changement de logique pour l'aménageur. La demande relève donc bien de la 


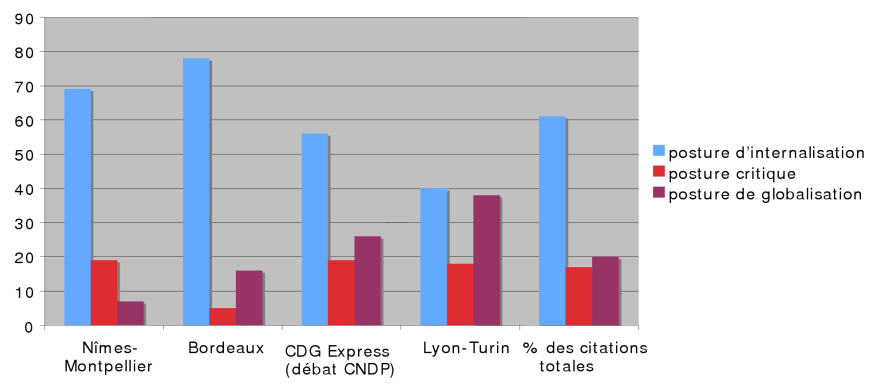

Fig. 1. Répartition des postures au sein de chaque cas étudié.

première posture, l'enjeu étant d'élargir le champ des considérations à prendre en compte selon une approche d'internalisation des effets jusque-là externes. Les oppositions restantes s'expriment alors sur l'insuffisance des évaluations réalisées, des normes retenues (bruit) ou des moyens alloués pour amoindrir les effets négatifs. L'enjeu pour le porteur du projet réside dans sa capacité à prendre la mesure du caractère structurant du projet pour les territoires concernés. L'infrastructure doit ainsi être pensée pour les espaces et les populations spécifiquement concernés et chercher à maximiser les opportunités locales.

Enfin, la troisième posture est également présente et regroupe les questionnements restants. Les thématiques : " articulation politique d'aménagement du territoire et politique des transports », « choix de société » (évolution des tendances générales de trafic et répartition modale, pourcentage des projets territoriaux locaux, intégrant le projet de transport), et une partie des questions de la thématique "équité, répartition » sont abordées selon cette posture globalisante. Sur ces thématiques, l'expression favorite est celle de l'argumentation sur un plan intellectuel, voire philosophique. Si les deux autres postures étaient fortement ancrées sur le projet en lui-même, les thématiques ici traitées appréhendent la dimension politique de ce type de projet, ouvrant les débats sur les politiques publiques d'infrastructures, d'économie et d'aménagement du territoire et d'urbanisme. Les modes de consommation et de développement, les répercussions sociales de la politique des transports, l'environnement et, dans une certaine mesure, le développement durable sont questionnés. Ces thématiques sont ainsi marquées par un certain recul sur le plan de l'action, le débat se portant dans le monde des idées. Si l'aménageur n'est plus nécessairement le bon interlocuteur de ces interpellations, on peut néanmoins imaginer qu'il lui appartient de transmettre ces interrogations à une échelle politique pour une mise à l'agenda.

La répartition des postures au sein de chaque cas est illustrée par la figure ci-dessous.

L'ensemble des cas montre une prédominance de la posture d'internalisation.
$\mathrm{Si}$, dans trois cas sur quatre, la posture de globalisation est privilégiée par rapport à la posture critique, dans le cas de Nîmes-Montpellier, c'est au contraire celle-ci que l'on retrouve en bonne position du fait notamment d'une perception négative du projet par les populations locales, qui attendent dès lors de la part du maître d'ouvrage des compensations plus que des solutions. Dans cet état d'esprit, la posture de globalisation n'a pas trouvé d'écho dans le cadre du débat $(7 \%)$.

Concernant l'importance relative de la posture de globalisation dans le cas Lyon-Turin, on retiendra que ce projet cible spécifiquement le fret. De ce fait, il interroge directement les choix de mode de transport, qui plus est à une échelle intraeuropéenne, ce qui est propice à l'adoption d'une telle posture. Par ailleurs, le côté « invasif » du projet - il s'agit de traverser la montagne accentue cette prise de conscience globale.

La posture critique est bien présente sur le cas CDG Express pour les mêmes raisons que dans le cas NîmesMontpellier, bien que le contexte soit différent, les populations critiquant fortement le projet du fait que les bénéficiaires futurs n'appartiennent pas au territoire concerné.

Enfin, dans le cas de Bordeaux, la nature de la concertation et le type de projet sur lequel elle porte expliquent la part relativement élevée de la posture d'internalisation : le projet se situant dans un environnement très urbanisé, il ne s'agit donc pas de changer sa logique mais plutôt de l'intégrer au mieux. En revanche, même dans un tel contexte, la posture globalisante est loin d'être absente des débats.

\section{Les enjeux de gouvernance liés à l'utilisation des systèmes d'indicateurs}

Quelle que soit la "posture » à laquelle les expériences étudiées se rattachent, le lien entre production et utilisation des indicateurs renvoie à des enjeux de gouvernance. Nous proposons de regrouper ces enjeux en trois points, pour lesquels nous avons identifié des questions phare pour la conception de tels systèmes d'information.

1) L'analyse bibliographique a montré que, si les objectifs affichés des expériences étudiées restent relativement généraux - ils visent globalement l'équilibre général entre les différentes dimensions du développement durable , ceux-ci n'en restent pas moins nécessairement déclinés au stade de l'élaboration des systèmes d'indicateurs. Des choix, avec leur cortège de "renoncements ", sont alors faits, souvent de manière peu explicite. Or, ceux-ci peuvent être très différents d'une expérience à l'autre, en fonction notamment des groupes sociaux qui élaborent les systèmes d'indicateurs et des valeurs qu'ils défendent (experts du secteur des transports, environnementalistes, organismes internationaux, etc.). Les systèmes d'indicateurs s'inscrivent ainsi dans des mondes particuliers, des 
collectifs, qui possèdent leurs propres modes de fonctionnement et de régulation (prix, réglementations, choix stratégiques...). C'est dans ces cadres implicites que les indicateurs ont alors vocation à initier des démarches politiques ou pratiques pour orienter les politiques de transport, dont les champs sont limités par ces mêmes cadres. $C^{\prime}$ est également en référence à ces cadres que peuvent se justifier les choix (et les pertes correspondantes) orientant les systèmes d'indicateurs et notamment la hiérarchie des thématiques traitées. Concrètement, cela se traduit, pour un certain nombre d'expériences, par des choix privilégiant les leviers d'action traditionnels des politiques de transport : on ne se préoccupe que de ce que l'on peut dominer, suivre, grâce à des moyens que l'on sait pouvoir mobiliser.

La question de l'utilisation des indicateurs au sein des débats publics doit également être étudiée en tenant compte de ces cadres collectifs implicites, de leurs frontières et de leurs logiques : comment se définissent le ou les collectifs impliqués dans les débats, censés utiliser les indicateurs? Comment fonctionnent-ils? Les indicateurs mis en débat ont-ils un sens commun ou différent pour les collectifs en présence dans les débats? La confrontation de ces collectifs permet-elle de faire émerger de nouveaux indicateurs et, si oui, quels sens leur donner?

2) Le deuxième point concerne la question de la légitimité des mondes impliqués pour construire des systèmes d'indicateurs, que l'on soit dans la posture 1 , où cette question est abordée sous l'angle de la responsabilisation du secteur des transports vis-à-vis des problématiques environnementales et sociales auxquelles il est confronté, ou dans les postures 2 et 3 , où cette question est posée d'un point de vue externe au secteur des transports : jusqu'où le monde des transports peut-il décider lui-même de la manière d'intégrer et de prendre en compte les autres dimensions du développement durable?

Cette question de légitimité se retrouve également dès lors que l'on s'intéresse à l'utilisation des indicateurs. Au sein des débats publics, l'utilisation d'indicateurs dans les discours pose ainsi la question de «qui parle » et «à qui », avec quelle légitimité pour utiliser et mettre en débat des indicateurs?

3) Dans la construction de ces systèmes d'indicateurs, la place de la norme apparaît centrale pour aborder la question des limites du monde dans lequel s'inscrit chaque système d'indicateurs et celle de la place des politiques de transport au sein de ce monde (quel rapport de grandeur implicite existe-t-il entre la politique des transports et les thématiques du développement durable?). Différentes situations se rencontrent dans les expériences étudiées. La norme peut tout d'abord être posée ex ante (c'est le cas, par exemple, des normes environnementales qui assoient certaines indicateurs de performance) ; elle constitue alors soit un objectif des politiques de transport, soit une contrainte qui encadre leur action et leur développement (le deuxième cas est évidemment le plus fréquent). La norme peut également être identifiée non pas ex ante, mais par comparaison, dans les démarches conçues dans une perspective de benchmarking : il s'agit ici de pointer les marges de progrès des politiques de transport au regard $\mathrm{d}^{\prime}$ autres politiques de transport.

Cette question de la place de la norme est également centrale quand on s'intéresse à l'utilisation des indicateurs : comment le collectif qui débat produit et utilise les normes pour structurer son action? En quoi les débats publics modifient-ils ou remettent-ils en question les normes proposées au travers des indicateurs discutés? En quoi ces normes interagissent-elles sur la nature des débats et sur leur évolution? En quoi la place de la norme dans la phase de construction des indicateurs est-elle déterminante ou pas dans l'utilisation de ces indicateurs au sein des débats publics?

Il apparaît donc que la question de la gouvernance est centrale et commune à toutes les expériences étudiées, notamment lorsque l'on s'intéresse à l'utilisation potentielle de ces indicateurs dans un cadre décisionnel. Les questions de normes, de collectifs institués, de légitimité sont ainsi au cœur des conditions d'utilisation de ces indicateurs. À ce titre, la synthèse réalisée précédemment confirme à nos yeux l'intérêt de concevoir des systèmes d'information sur le développement durable qui soient modulaires, proposant des postures différentes de jugement de la politique des transports. Attaché à un contexte, un ensemble d'indicateurs doit refléter la légitimité et la responsabilité de ses utilisateurs à l'égard des questionnements en matière de développement durable. En dernier ressort, il convient de reconnaître aux indicateurs du développement durable, non pas une fonction univoque d'évaluation, mais le statut d'instruments de négociation entre les différentes parties prenantes. Notre cadre de lecture a donc fait émerger le discours derrière l'instrument d'évaluation que constitue un système d'indicateurs. Mais la typologie de postures a également montré son intérêt pour décrypter les discours échangés au cours des débats publics et des concertations. Si ces procédures de participation du public sont aujourd'hui en voie de généralisation dans le domaine des politiques publiques, nombreux sont ceux qui s'interrogent sur leur utilité concrète en matière décisionnelle. Nous pensons que l'exploitation des débats proposée ici permet de rendre compte de manière formelle de la teneur de ces échanges. La structuration des arguments sous la forme d'indicateurs de la parole constituerait une grille d'évaluation des projets d'infrastructure qui permettrait de pérenniser certains apports de ces procédures de concertation.

\section{Références}

AEE, 2000. Est-ce la bonne route? Indicateurs d'intégration transport et environnement dans l'UE : TERM 2000. Document de synthèse. Rapport, AEE, Copenhague. 
Barret, J., Scott, A., Vallack, H., 2001. The Ecological Footprint of Passenger Transport in Merseyside. Rapport pour le compte de Merseytravel, Stockholm Environment Institute/Sustainable Steps Environmental Consultants, York (http://www. merseytravel.gov.uk/pdf/EFofPassengerTransport.pdf).

Bell, K., 1999. Environmental Performance Indicators: Proposals for Indicators of the Environmental Effects of Transport. Report of the Ministry for the Environment New Zealand, Wellington.

Borken J., 2003. Indicators for sustainable mobility: a policy oriented approach, in Joumard, R. (Ed.), Environnement $\mathcal{E}$ transports : [actes du] $1^{\text {er }}$ colloque [international Environnement $\mathcal{E}$ transports], Avignon, 19-20 juin 2003, Arcueil, INRETS.

Braat L., 1991. The predictive meaning of sustainable indicators, in Kuik, O., Verbruggen, H. (Eds), Search of Indicators of Sustainable Development, Dordrecht, Kluwer Academic Publisher, 57-70.

CDD [Commission du développement durable du Conseil économique et social des Nations unies], 2001a. Informations et institutions pour la prise de décision. Rapport du secrétaire général.

CDD, 2001b. Transport and Sustainable Development in the ECE Region. Report 9th session, 16-27 avril, New York.

CDD, 2001c. Indicators of Sustainable Development: Guidelines and Methodologie, New York, United Nations.

CEMT [Conférence européenne des ministres des transports], 1998. L'Évaluation environnementale stratégique dans le secteur des transports, Paris, OCDE.

CEMT, 2003a. L'Acquis CEMT : 1953 -2003, Paris, CEMT/OCDE.

CEMT, 2003b. Cinquante ans de politique des transports : succès, échecs et nouveaux défis, Paris, OCDE.

CEMT, 2005. 50 ans de recherche en économie des transports : l'expérience acquise et les grands enjeux. Rapports introductifs et synthèse des discussions, $16^{e}$ symposium international sur la théorie et la pratique dans l'économie des transports, Budapest 29-31 octobre 2003, Paris, CEMT/OCDE.

Reçu le 11 septembre 2007. Accepté le 30 juin 2009.
OCDE, 2001a. Indicateurs de performance dans le secteur routier, Paris, OCDE.

OCDE, 2001b. Évaluer les avantages des transports, Paris, OCDE.

OCDE, 2001c. TEV : transports écologiquement viables. Rapport de synthèse de la commission Transport du Comité des politiques d'environnement de l'OCDE préparé pour la conférence de Vienne en octobre 2000.

OCDE, 2003. Lignes directrices de l'OCDE sur les transports écologiquement viables, Paris, OCDE (http://browse.oecdbookshop. org/oecd/pdfs/browseit/9702192E.PDF).

PROPOLIS, 2004. Planning and Research of Policies for Land Use and Transport for Increasing Urban Sustainibility. Projet dans le cadre du $5^{\mathrm{e}}$ PCRD (programme-cadre de recherche et de développement technologique de la Commission européenne).

Rahman, A., Van Grol, R., 2005. Sustainable Mobility: Policy Measures and Assessment. Consortium dirigé par RAND Europe, Adnan et Rik pour le $5^{\mathrm{e}}$ PCRD.

Stott, H., 2002. Monitoring Progress Towards a Sustainable New Zealand. Statistics New Zealand, Wellington.

Transport Statistics Personal Travel UK, 2004. How to Monitor Indicators in Local Transport Plans and Annual Progress Reports - 2004 update. Department for Transport, London.

Verbruggen, H., Kuik, O., 1991. In Search of Indicators of Sustainable Development, Dordrecht, Kluwer Academic Publishers.

Verry, D., Nicolas, J.-P., 2005. Indicateurs de mobilité durable : de l'état de l'art à la définition des indicateurs dans le projet Simbad. Rapport du Laboratoire d'économie des transports pour le compte de la DRAST et de l'ADEME dans le cadre du groupe $11 \mathrm{du}$ PREDIT, Vaulx-en-Velin. 